\begin{tabular}{|c|l|}
\hline Title & Mode conversion effects of phonons on A nderson local ization \\
\hline Author(s) & Yoshihiro, Tatsuya; Nishiguchi, Norihiko \\
\hline Citation & $\begin{array}{l}\text { Physical Review B, 100(23), 235441 } \\
\text { https://doi.org/10.1103/PhysRevB.100.235441 }\end{array}$ \\
\hline Issue Date & 2019-12-23 \\
\hline Doc URL & http://hdl.handle.net/2115/76615 \\
\hline Rights & Copyright 2019 by The A merican Physical Society. \\
\hline Type & article \\
\hline File Information & PhysRevB.100.235441.pdf \\
\hline
\end{tabular}

Instructions for use 


\title{
Mode-conversion effects of phonons on Anderson localization
}

\author{
Tatsuya Yoshihiro $\oplus^{1,2, *}$ and Norihiko Nishiguchi ${ }^{3}$ \\ ${ }^{1}$ Graduate School of Engineering, Department of Applied Physics, Hokkaido University, Sapporo 060-8628, Japan \\ ${ }^{2}$ Frontier Core Technology Laboratories FUJIFILM Corporation 577, Ushijima, Kaisei-Machi, \\ Ashigarakami-gun Kanagawa 258-8577, Japan \\ ${ }^{3}$ Faculty of Engineering, Division of Applied Physics, Hokkaido University, Sapporo 060-8628, Japan
}

(Received 18 April 2019; revised manuscript received 13 October 2019; published 23 December 2019)

\begin{abstract}
Numerically investigating mode-conversion effects of phonons on Anderson localization in random superlattices, we make it clear the changes in localization properties when the LA and TA phonons which independently show Anderson localization for the normal incidence interact as the incident angles increase. The mode conversions which occur for oblique propagation at small incident angles induce collaboration among the phonons, giving rise to extension of the localization length or delocalization, depending on the system size. On the other hand, at large incident angles, the transmission decay becomes larger than for the normal incidence. In contrast to the small incident angles, the transmission decay and transmission fluctuations become independent of the incident phonon modes and show the frequency dependences that are not predictable from those at the small incident angles, evidencing that the coupled modes of phonons are formed by the mode conversions.
\end{abstract}

DOI: 10.1103/PhysRevB.100.235441

\section{INTRODUCTION}

Anderson localization is a phenomenon which is common to electrons and classical waves such as light and sound waves in disordered media [1-12]. It is known that phonons will not propagate through such a medium containing a large amount of randomness. Anderson localization arises from interference among scattered phonons owing to the randomness, and then the phenomenon is apt to take place at low dimensions [13]. In particular, in one dimension any disorder is strong enough to induce the exponential localization of eigenmodes. In previous studies [14-16], Anderson localization of phonons in randomly layered superlattices are investigated, using the transfer-matrix method and Green's functions. The random superlattices (RSL's) are the multilayered system where two kinds of unit layers of dissimilar materials are randomly stacked. The relevant quantity to characterize the phonon propagation is the transmission rates. The frequency dependence of transmission rates exhibits a fine spiky structure specific to the particular realization of disorder of constituent layers. The fine structures are smeared out by the ensemble average over the possible configurations of the constituting layers, and the resultant global structures exhibit the transmission decay due to Anderson localization in the wide frequency region and some peaks due to the resonance transmission where the phonon wavelength matches the thicknesses of the unit layers. As for the fluctuations of transmission rates, there is a universal relation for the transmission fluctuations versus the average transmission irrespective of the kinds of randomness.

From the transmission properties of phonons, the onedimensional RSL's are expected to be a thermal device to

*Corresponding author: tatsuya.yoshihiro@fujifilm.com control heat conduction [17-21]. The transmission properties of phonons in the RSL's stated above are obtained for single mode phonons when the phonons impinge normally on the RSL's. Supposing that the RSL's are connected to a heat bath, most of the phonons will impinge on the RSL's at oblique angles. For oblique phonon incidence, the longitudinal acoustic (LA) phonons and transverse acoustic (TA) phonons are no longer independent due to the mode conversions occurring at the interfaces of RSL's. Then the LA phonons and TA ones coexist in each layer of the RSL's, irrespective of the incident phonon modes. Because the LA phonons have a sound velocity larger than that of the TA phonons, their wave vectors are not parallel in the layers due to Snell's law and the phases develop individually with propagation, modifying the interference conditions of phonons. Then Anderson localization for oblique phonon propagation is expected to be substantially different from those in case of the normal phonon incidence.

Here we guess the effects of the mode conversions on the phonon propagation in the RSL's. Suppose that the LA phonons are localized and the TA phonons are extended in a finite RSL at the same frequency. We expect that phonon energy input by the LA phonons is possible to be transmitted as the TA phonons, and vice versa. If so, the effective phonon transmission will not decay thanks to the collaboration of phonons. On the other hand, the mode conversions might form coupled phonon modes peculiar to the system. If the phonon modes exist in the RSL's, contrary to the above speculation, the phonons will decay due to Anderson localization because the coherence of phonons should be maintained as long as in the absence of inelastic scattering of phonons. These speculations lead to the opposite transmission properties of phonons in the RSL's. If the former effects violate Anderson localization of phonons, the usage of random superlattice might not be appropriate for controlling phonon propagation. 


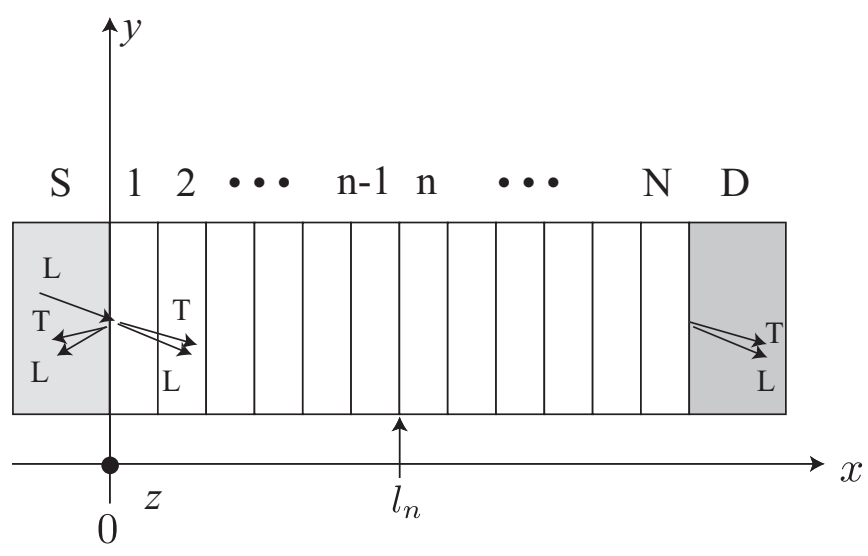

FIG. 1. Model of a random superlattice made of $N$ layers sandwiched between the substrate(S) and detection layer(D). Arrows labeled by $\mathrm{L}$ and $\mathrm{T}$ represent the longitudinal and transverse acoustic phonon propagations.

Thus, we are interested in the phonon transmission in the RLSs at oblique angles from not only for scientific reasons but also for engineering ones.

The purpose of this work is to elucidate the effects of mode conversions of phonons on Anderson localization. In Sec. II we model the RSL's and give an equation of motion in the system. We introduce in Sec. III the transfer-matrix method for describing phonon propagation in the RSL's, and define the transmission rate using the Poynting vector of phonons. In Sec. 4 we illustrate the transmission rates, Lyapunov exponents, and transmission fluctuations for GaAs/AlAs RSL's, varying the incident angles of phonons. We investigate the changes in characteristics of phonon modes with respect to the incident angles. Summary and discussion are given in Sec. V.

\section{MODEL}

We introduce the RSL's that are constructed by stacking two kinds of single layers at random with equal occurrence probabilities, i.e., 0.5 as illustrated in Fig. 1. Each layer is composed of a material A or B with a thickness $d_{\mathrm{A}}$ or $d_{\mathrm{B}}$. The total number of layers is $N$, and the RSL is sandwiched between the substrate(S) and detection layer(D), both of which are composed of material A. Considering that phonons with wavelength larger than the unit layers exhibit characteristic transmission properties of Anderson localization [15], we may simulate the RSL's using an elastic continuum model rather than microscopic models $[11,12]$ with random atomic configurations. We also assume isotropic elasticity for the constituting materials, avoiding complexities arising from anisotropic elasticity such as phonon focusing effects.

We put the $x$ axis to be parallel to the normal direction of interfaces in the RSL and assume the layers to be uniform in the $y-z$ plane. Here we define the position of the interface $l_{n}$ separating $(n-1)$ th and $n$th layers by

$$
l_{n}=\sum_{i=0}^{n-1} d_{i}
$$

where $l_{1}=d_{0}=0$.
The equation of motion in each layer including the substrate and detection layer is

$$
\rho_{\{n, S, D\}} \ddot{u}_{i}=\partial_{j} C_{\{n, S, D\}, i j k l} \partial_{k} u_{l}, \quad(i, j, k, l=x, y, z)
$$

where $u_{i}$ is an $i$ th component of the displacement vector, $\rho_{\{n, S, D\}}$ and $C_{\{n, S, D\}, i j k l}$ are the mass density and the stiffness tensor of the corresponding layer. A sum over repeated indices is assumed in Eq. (2).

\section{FORMALISM}

Transmission properties of phonons depend on the polarizations for oblique propagation in the layered structures. One of the two TA phonon modes, which has the polarization parallel to the interface, does not give rise to mode conversions at the interfaces of RSL's. Then, the transmission properties in the RSL's are essentially the same as those of single mode phonons studied in Refs. [15,16]. Another TA mode has the polarization in the sagittal plane formed by the wave vector $\mathbf{q}$ and the superlattice axis. Since the LA phonons have the polarization in the sagittal plane, the mode conversions between the TA and LA phonons take place at the interfaces. In the present system, we assign the $x-y$ plane to be the sagittal plane and the $z$ axis to be perpendicular to the plane. Putting the $z$ component of a wave vector to be $q_{z}=0$, we investigate the mode-conversion effects of phonons propagating in the sagittal plane. The relevant LA and TA phonons have the displacement vectors in the $x-y$ plane as $\mathbf{u}=\left(u_{x}, u_{y}, 0\right)$.

We use the transfer-matrix method to examine phonon propagation in the RSL's. We express the sound velocities and wave vectors of phonons in an $n$th layer, the substrate and the detection layer as $c_{\{n, S, D\},\{L, T\}}$ and $\mathbf{q}_{\{n, S, D\},\{L, T\}}$, and assume the linear dispersion relations

$$
\omega=\left|\mathbf{q}_{\{n, S, D\},\{L, T\}}\right| c_{\{n, S, D\},\{L, T\}},
$$

where the subscripts $L$ and $T$ stand for the LA and TA modes. The wave vectors have the same $y$ component in all the layers given by

$$
q_{y}=\frac{\omega}{c_{\{n, S, D\},\{L, T\}}} \sin \theta_{\{n, S, D\},\{L, T\}},
$$

due to Snell's law. Here $\theta_{\{n, S, D\},\{L, T\}}$ is the angle of the wave vector of phonons in each layer measured from the $x$ axis. The wave number in the $x$ direction of phonons propagating toward the $x_{+}$direction yields

$$
q_{\{n, S, D\},\{L, T\}}=\frac{\omega}{c_{\{n, S, D\},\{L, T\}}} \cos \theta_{\{n, S, D\},\{L, T\}} .
$$

Putting the wave vectors of transmitted and reflected phonons as $\mathbf{q}^{+}$and $\mathbf{q}^{-}$as

$$
\mathbf{q}_{\{n, S, D\},\{L, T\}}^{ \pm}=\left( \pm q_{\{n, S, D\},\{L, T\}}, q_{y}, 0\right)
$$

the corresponding polarization vectors are given by

$$
\begin{gathered}
\mathbf{e}_{\{n, S, D\}, L}^{ \pm}=\mathbf{q}_{\{n, S, D\}, L}^{ \pm} /\left|\mathbf{q}_{\{n, S, D\}, L}^{ \pm}\right| \\
\mathbf{e}_{\{n, S, D\}, T}^{ \pm}=\mathbf{q}_{\{n, S, D\}, T}^{ \pm} /\left|\mathbf{q}_{\{n, S, D\}, T}^{ \pm}\right| \times \mathbf{e}_{z} .
\end{gathered}
$$

Using them, the displacement vector of phonons in the $n$th layer becomes

$$
\begin{aligned}
\mathbf{u}_{n}= & \left(\mathbf{e}_{n, L}^{+} a_{n} e^{i q_{n, L} x}+\mathbf{e}_{n, L}^{-} b_{n} e^{-i q_{n, L} x}\right) e^{i q_{y} y} \\
& +\left(\mathbf{e}_{n, T}^{+} c_{n} e^{i q_{n, T} x}+\mathbf{e}_{n, T}^{-} d_{n} e^{-i q_{n, T} x}\right) e^{i q_{y} y},
\end{aligned}
$$


where $a_{n} b_{n} c_{n}$ and $d_{n}$ are coefficients of transmitted and reflected phonons. We express the displacement vectors in the substrate and detection layer in the same way;

$$
\begin{gathered}
\mathbf{u}_{S}=\left(\mathbf{e}_{S, L}^{+} a_{S} e^{i q_{S, L} x}+\mathbf{e}_{S, L}^{-} b_{S} e^{-i q_{S, L} x}\right) e^{i q_{y} y} \\
+\left(\mathbf{e}_{S, T}^{+} c_{S} e^{i q_{S, T} x}+\mathbf{e}_{S, T}^{-} d_{S} e^{-i q_{S, T} x}\right) e^{i q_{y} y} \\
\mathbf{u}_{D}=\mathbf{e}_{D, L}^{+} t_{L} e^{i q_{D, L}\left(x-l_{D}\right)} e^{i q_{y} y}+\mathbf{e}_{D, T}^{+} t_{T} e^{i q_{D, T}\left(x-l_{D}\right)} e^{i q_{y} y},
\end{gathered}
$$

where $a_{S}$ and $c_{S}$ are the coefficients of incident LA and TA phonons, and $b_{S}$ and $d_{S}$ are those of reflected phonons in the substrate. $t_{L}$ and $t_{T}$ are the coefficients of transmitted LA and TA phonons in the detection layer. Here $l_{D}=l_{N+1}$, which denotes the interface between the RSL and D-layer. The displacement vectors (8), (9), and (10) satisfy Eq. (2) in each layer.

The $x$ and $y$ components of a stress vector acting on the $y-z$ plane yield

$$
\sigma_{\{n, S, D\}, x X}=C_{\{n, S, D\}, 11} \partial_{x} u_{\{n, S, D\}, x}+C_{\{n, S, D\}, 12} \partial_{y} u_{\{n, S, D\}, y}
$$

$$
\sigma_{\{n, S, D\}, y X}=C_{\{n, S, D\}, 44} \partial_{x} u_{\{n, S, D\}, y}+C_{\{n, S, D\}, 44} \partial_{y} u_{\{n, S, D\}, x},
$$

where the stiffness tensors are substituted by the stiffness constants in Voigt notation for cubic materials.

Considering that the displacement vectors and the stress vectors in adjoining layers match at their interface, we obtain the following equation about the coefficients of the incident phonons and the transmitted phonons

$$
\left(a_{S}, b_{S}, c_{S}, d_{S}\right)^{T}=W\left(t_{L}, 0, t_{T}, 0\right)^{T},
$$

where $W$ is the matrix defined by

$$
W \equiv \Lambda_{S}^{-1}\left[\prod_{n=1}^{N} \Lambda_{n}(0) \Lambda_{n}^{-1}\left(d_{n}\right)\right] \Lambda_{D} .
$$

Here $\Lambda_{n}\left(d_{n}\right), \Lambda_{S}$, and $\Lambda_{D}$ are matrices, which are given in Appendix.

Putting $w_{i j}$ as a matrix element of $W$, the transmission coefficients $t_{L}$ and $t_{T}$ are related to $a_{S}$ and $c_{S}$ as

$$
\begin{aligned}
\left(\begin{array}{c}
t_{L} \\
t_{T}
\end{array}\right)= & \frac{1}{w_{11} w_{33}-w_{13} w_{31}}\left(\begin{array}{c}
w_{33} \\
-w_{31}
\end{array}\right) a_{S} \\
& +\frac{1}{w_{11} w_{33}-w_{13} w_{31}}\left(\begin{array}{c}
-w_{13} \\
w_{11}
\end{array}\right) c_{S} .
\end{aligned}
$$

Thus, even if the incident phonons were either the LA phonons $\left(a_{S}=1, c_{S}=0\right)$ or TA ones $\left(a_{S}=0, c_{S}=1\right)$, the transmitted phonons are composed of them. This is the case for the reflection. The reflection coefficients $b_{S}$ and $d_{S}$ in the substrate are given by

$$
\begin{aligned}
\left(\begin{array}{l}
b_{S} \\
d_{S}
\end{array}\right)= & \frac{1}{w_{11} w_{33}-w_{13} w_{31}}\left(\begin{array}{l}
w_{21} w_{33}-w_{23} w_{31} \\
w_{41} w_{33}-w_{43} w_{31}
\end{array}\right) a_{S} \\
& +\frac{1}{w_{11} w_{33}-w_{13} w_{31}}\left(\begin{array}{l}
-w_{21} w_{13}+w_{23} w_{11} \\
-w_{41} w_{13}+w_{43} w_{11}
\end{array}\right) c_{S} .
\end{aligned}
$$

Here we define the transmission rate using the Poynting vector of phonons. From the equation of continuity of elastic energy, we define an $i$ th component of the Poynting vector as $J_{i} \equiv-\dot{u}_{j} \sigma_{i j}$. The $x$ component of Poynting vector for incident phonons having $\omega$ and $q_{y}$ becomes

$$
J_{S, x}\left(\omega, q_{y}\right)=\left|a_{S}\right|^{2} \omega^{2} \frac{C_{S, 11}}{c_{S, L}} \cos \theta_{S, L}+\left|c_{S}\right|^{2} \omega^{2} \frac{C_{S, 44}}{c_{S, T}} \cos \theta_{S, T},
$$

and that of transmitted phonons in the detection layer is

$J_{D, x}\left(\omega, q_{y}\right)=\left|t_{L}\right|^{2} \omega^{2} \frac{C_{D, 11}}{c_{D, L}} \cos \theta_{D, L}+\left|t_{T}\right|^{2} \omega^{2} \frac{C_{D, 44}}{c_{D, T}} \cos \theta_{D, T}$.

We define the transmission rate $T\left(\omega, q_{y}\right)$ by the ratio of these quantities

$$
T\left(\omega, q_{y}\right) \equiv \frac{J_{D, x}\left(\omega, q_{y}\right)}{J_{S, x}\left(\omega, q_{y}\right)} .
$$

Here we introduce $T_{l m}$ that is defined by the ratio of the Poynting vector of $m$-mode phonons in Eq. (18) to that of the incident $l$-mode phonons in Eq. (17). In terms of them, the transmission rates are divided into two parts as $T_{L}=$ $T_{L L}+T_{L T}$ for the LA phonon incidence and $T_{T}=T_{T L}+T_{T T}$ for the TA phonon incidence. The contributions of them will be discussed below. We also introduce the reflectances $R_{l m}$ defined by the ratio of the Poynting vector of reflected $m$ mode phonons to that of incident $l$-mode phonons.

\section{RESULTS}

We illustrate the phonon transmission properties of the RSL's made of GaAs and AlAs which are referred to as the materials $\mathrm{A}$ and $\mathrm{B}$ in the rest of the paper. The unit layers have the same thickness $d_{\mathrm{A}}=d_{\mathrm{B}}=3.4 \mathrm{~nm}$ so that the size of RSL's can be fixed for a given $N$. We introduce the effective Lamè constants $\lambda_{\mathrm{A}, \mathrm{B}}$ and $\mu_{\mathrm{A}, \mathrm{B}}$ which are estimated from the sound velocities averaged over the angles in the $x-y$ plane; $\lambda_{\mathrm{A}}=4.351 \times 10^{11} \mathrm{dyn} / \mathrm{cm}^{2}, \mu_{\mathrm{A}}=$ $4.490 \times 10^{11} \mathrm{dyn} / \mathrm{cm}^{2}, \lambda_{\mathrm{B}}=4.652 \times 10^{11} \mathrm{dyn} / \mathrm{cm}^{2}, \mu_{\mathrm{B}}=$ $4.419 \times 10^{11} \mathrm{dyn} / \mathrm{cm}^{2}$. Their mass densities are $\rho_{\mathrm{A}}=$ $5.36 \mathrm{~g} \mathrm{~cm}^{-3}$ and $\rho_{\mathrm{B}}=3.76 \mathrm{~g} \mathrm{~cm}^{-3}$. The material parameters used for calculations are quoted from Refs. [22,23] for GaAs and from Refs. [24,25] for AlAs.

Using the material parameters, we have the sound velocities of $\mathrm{LA}$ and $\mathrm{TA}$ phonons as $c_{\mathrm{A}, \mathrm{L}}=$ $4.987 \times 10^{3} \mathrm{~m} / \mathrm{s}, c_{\mathrm{A}, \mathrm{T}}=2.894 \times 10^{3} \mathrm{~m} / \mathrm{s}, c_{\mathrm{B}, \mathrm{L}}=5.990 \times$

$10^{3} \mathrm{~m} / \mathrm{s}, c_{\mathrm{B}, \mathrm{T}}=3.428 \times 10^{3} \mathrm{~m} / \mathrm{s}$. Since $c_{A, L} / c_{B, L}<1$, the angles of incident phonons $\theta_{S, L}$ and $\theta_{S, T}$ must be within the following ranges from Eq. (3),

$$
\begin{aligned}
& 0 \leqslant \theta_{S, L}<\sin ^{-1} \frac{c_{A, L}}{c_{B, L}}=56.3^{\circ} \\
& 0 \leqslant \theta_{S, T}<\sin ^{-1} \frac{c_{A, T}}{c_{B, L}}=29.0^{\circ} .
\end{aligned}
$$

\section{A. Transmission rates}

For the oblique phonon incidences, the LA phonons and TA ones that have the same frequency and wave number in the $y$ direction $\left(\omega, q_{y}\right)$ as the incident phonons coexist in all the layers of the RSL's, irrespective of the incident phonon 

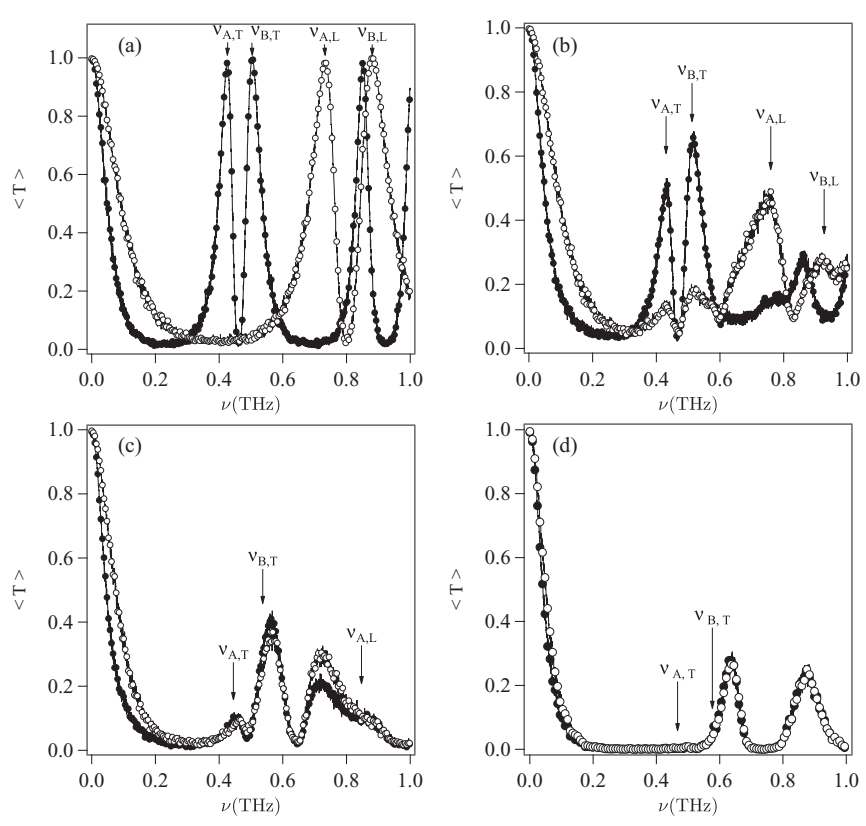

FIG. 2. The averaged transmission rates versus frequency in GaAs/AlAs RSL's. The number of layers is $N=1000$, and 300 samples of RSL's are used for ensemble average of the transmission rates. The incident angles are (a) $\theta_{S, L}=0^{\circ}, \theta_{S, T}=0^{\circ}$, (b) $\theta_{S, L}=$ $15.0^{\circ}, \theta_{S, T}=8.6^{\circ}$, (c) $\theta_{S, L}=30.0^{\circ}, \theta_{S, T}=16.9^{\circ}$ and (d) $\theta_{S, L}=$ $45.0^{\circ}, \theta_{S, T}=24.2^{\circ}$. The solid lines with open circles denote $\langle T\rangle$ for the LA phonon incidence $\left(a_{S}=1, c_{S}=0\right)$, and the solid lines with solid circles denote $\langle T\rangle$ for the TA phonon incidence $\left(a_{S}=0, c_{S}=\right.$ 1). $v_{A, L}, v_{A, T}, v_{B, L}$, and $v_{B, T}$ are the resonance frequencies of LA and TA phonons in the unit layers of $\mathrm{A}$ and $\mathrm{B}$.

modes. However, the resultant transmission rates depend on the incident phonon modes. Then, we examine the transmission rates versus frequency for single mode phonon incidences at four pairs of incident angles $\theta_{S, L}$ and $\theta_{S, T}$ which satisfy the Snell's law; $q_{y}=\omega \sin \theta_{S, L} / c_{S, L}=\omega \sin \theta_{S, T} / c_{S, T}$.

Figure 2 shows the averaged transmission rates $\langle T\rangle$ versus frequency for the LA phonon incidence $\left(a_{S}=1, c_{S}=0\right)$ and that for the TA phonon incidence $\left(a_{S}=0, c_{S}=1\right)$ in the RSL's of $N=1000$ layers. The ensemble averages of the transmission rates are taken over 300 different configurations of unit layers. The data are shown at the four pair of incidence angles $\theta_{S, L}$ and $\theta_{S, T}$ ranging from the normal incidence to $\theta_{S, L}=45^{\circ}$ for the LA phonon incidence and to $\theta_{S, T}=24.2^{\circ}$ for the TA phonon incidence. The four pairs of incident angles $\theta_{S, L}$ and $\theta_{S, T}$ are given in the figure caption.

In case of the normal incidence of phonons [Fig. 2(a)], the LA and TA phonons propagate independently in the RSL's without mode conversions. Their averaged transmission rates individually show decay due to Anderson localization and peaks denoted by arrows owing to the resonance transmission where the wavelength matches the thicknesses of unit layers. The resonance frequencies are given by $[15,16]$

$$
v_{\{\mathrm{A}, \mathrm{B}\},\{\mathrm{L}, \mathrm{T}\}}=\frac{c_{\{\mathrm{A}, \mathrm{B}\},\{\mathrm{L}, \mathrm{T}\}}}{d_{\{\mathrm{A}, \mathrm{B}\}}} .
$$

Figure 2(b) shows $\langle T\rangle$ versus frequency at $\theta_{S, L}=15.0^{\circ}$ and $\theta_{S, T}=8.6^{\circ}$. The incident phonons are split into the LA and TA phonons having the same $q_{y}$. Like the normal incidence of
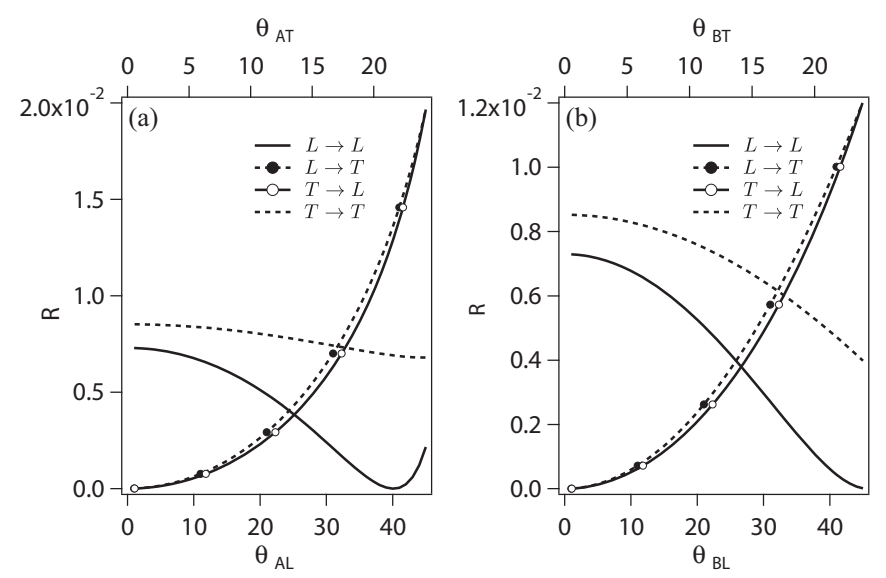

FIG. 3. Reflectance of the LA and TA phonons impinging on a single interface between A and B layers versus incident angles; (a) reflectance of the incident phonons in the A layer and (b) reflectance of the incident phonons in the B layer. The mode conversions become dominant for $\theta_{\{A, B\}, L}>25^{\circ}$ or $\theta_{\{A, B\}, T}>17^{\circ}$.

phonons shown in Fig. 2(a), $\langle T\rangle$ exhibits the decay and peaks distinctly for the LA and TA phonon incidences. Although the frequency dependences of $\langle T\rangle$ resemble those for the normal incidence, the transmission peaks shift to the higher frequency region as

$$
v_{\{\mathrm{A}, \mathrm{B}\},\{\mathrm{L}, \mathrm{T}\}}=\frac{c_{\{\mathrm{A}, \mathrm{B}\},\{\mathrm{L}, \mathrm{T}\}}}{d_{\{\mathrm{A}, \mathrm{B}\}} \cos \theta_{L, T}}
$$

because the wave number in the $x$ direction decreases as the incident angle increases. It is found that the transmission peaks are obviously smaller than those of the normal incidence. It is attributed to that phonons converted from the incident phonons do not simultaneously satisfy the resonance conditions of the incident phonons.

We also find that the transmission rates at the valleys are larger than those in Fig. 2(a) as if they are partly compensated by the transmission peaks of the converted phonons. It is considered that the converted phonons that satisfy the resonance conditions raise the total transmission rates.

Figure 2(c) shows $\langle T\rangle$ versus frequency at (c) $\theta_{S, L}=30.0^{\circ}$ and $\theta_{S, T}=16.9^{\circ}$. Remarkably, $\langle T\rangle$ are almost independent of the incident phonon modes. The transmission peaks of both cases appear at $v_{A, T}$ and $v_{B, T}$, and there is also a bump at $v_{\mathrm{A}, \mathrm{L}}$. In addition to the peaks, there is a peak between $\nu_{\mathrm{B}, \mathrm{T}}$ and $\nu_{\mathrm{A}, \mathrm{L}}$ which is not predicted by Eq. (23). The tendency becomes more apparent for $\theta_{S, L}=45.0^{\circ}$ and $\theta_{S, T}=24.2^{\circ}$ in Fig. 2(d). $\langle T\rangle$ for the LA and TA phonon incidences coincide well in the entire frequency region, and the magnitudes decrease more than Fig. 2(c). The frequencies of the two major peaks do not agree with Eq. (23) at all.

In order to investigate the transitional characteristics of $\langle T\rangle$ with increasing incident angles, we examine the incident angle dependence of reflectance at a single interface between the materials $\mathrm{A}$ and $\mathrm{B}$ since phonon backscattering is responsible for Anderson localization. Figure 3 shows the reflectance (a) when phonons in the A-layer impinges on the interface and (b) when phonons in the B-layer impinges on the interface. At the small incident angles, the mode 


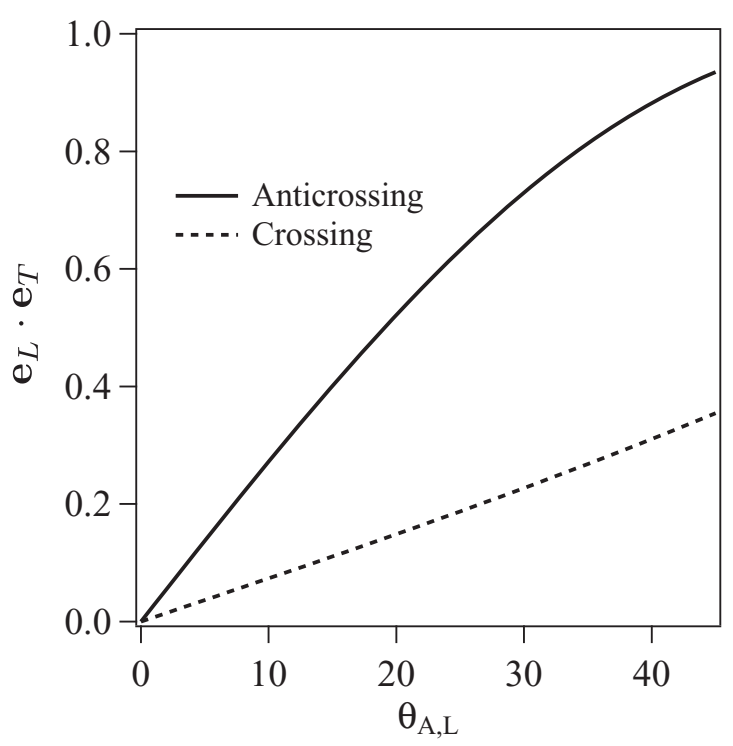

FIG. 4. The scalar products of the polarization vectors given by Eqs. (24) and (25) versus the propagation angle of LA phonons in a layer made of material A. The propagation angle of TA phonons is estimated from Snell's law. The results agree well those for a layer of material $\mathrm{B}$ in the entire angle region because the ratio of sound velocities $c_{A, L} / c_{A, T} \approx c_{B, L} / c_{B, T}$. The polarization vectors of transmitted and backscattered phonons (solid line) become parallel as the propagation angle increases in contrast to the phonons propagating in the same direction (dashed line).

conversions do not substantially take place. As a consequence, the phonon propagation in the RSL's will maintain their characteristics of the incident phonons. In contrast, the mode conversions become significant at large incident angles, in particular for $\theta_{L}>25^{\circ}$ or $\theta_{T}>16^{\circ}$. In the RSL's, the alternate exchanges of phonon modes occur repeatedly at all the interfaces, and the transmitted and backscattered phonons of different modes having wave vectors $\mathbf{q}_{n, L}^{ \pm}$and $\mathbf{q}_{n, T}^{\mp}$ encounter within every layer.

The polarization vectors of LA and TA phonons are perpendicular when the phonons propagate normally. For oblique propagating phonons, their polarization vectors will not be perpendicular but tend to be parallel at large angles of propagation. It is remarkable for the transmitted and backscattered phonons. The scalar product of the polarization vectors of the phonons becomes

$$
\mathbf{e}_{L(T)}^{ \pm} \cdot \mathbf{e}_{T(L)}^{\mp}=\sin \left(\theta_{n, L}+\theta_{n, T}\right)
$$

and for phonons having wave vectors $\mathbf{q}_{n, L}^{ \pm}$and $\mathbf{q}_{n, T}^{ \pm}$

$$
\mathbf{e}_{L(T)}^{ \pm} \cdot \mathbf{e}_{T(L)}^{ \pm}=\sin \left(\theta_{n, L}-\theta_{n, T}\right) .
$$

Figure 4 shows the scalar products of polarization vectors for the two cases in a layer of material A. The results agree well with those for a layer of material $\mathrm{B}$ in the entire angle region because the ratio of sound velocities $c_{A, L} / c_{A, T} \approx c_{B, L} / c_{B, T}$. The polarization vectors of transmitted and backscattered phonons are found to become parallel as the propagation angle increases. Considering that the phonons have almost the same polarization characters, we can expect coupling between the phonons at large incident angles. Actually such coupled
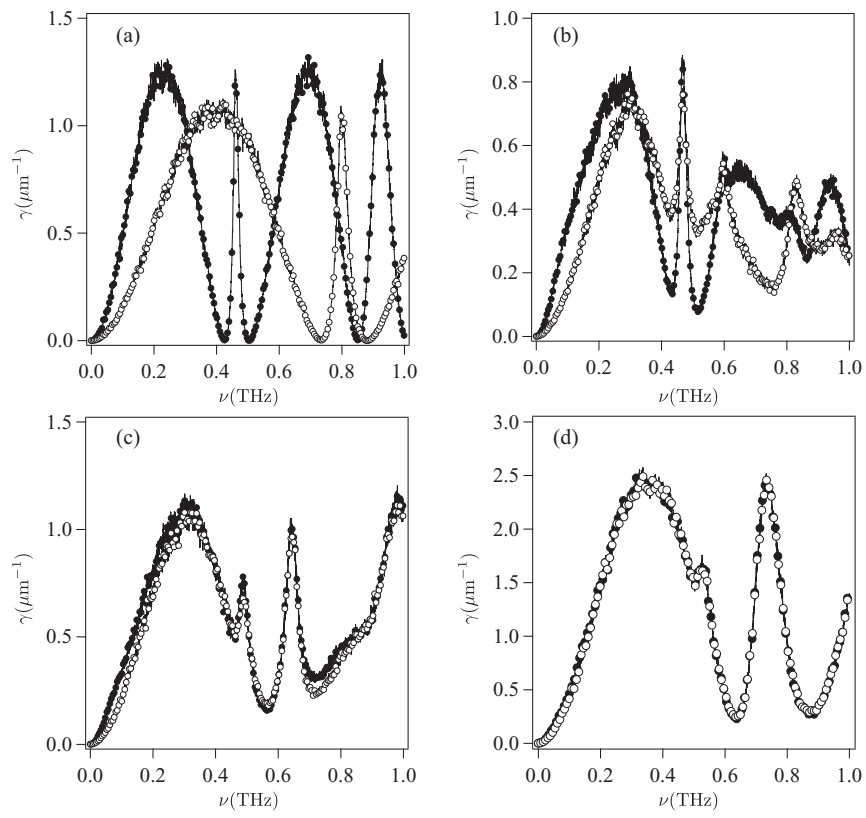

FIG. 5. The Lyapunov exponents versus frequency for the same four pairs of incident angles as Fig. 2. The solid lines with open (solid) circles indicates the Lyapunov exponents for the incident LA (TA) phonons.

phonon modes are found in a periodic superlattice for oblique phonon incidences [26]. The coupled phonon modes give rise to phonon stop bands inside the folded Brillouin zone as well as at the zone center and boundary. Assuming that the coupled phonon modes are supported in the RSL's, it is understandable that the transmission rates become insensitive to the incident phonon modes since they just are a portion of the coupled modes, and the coupled mode will have the resonance frequencies peculiar to the modes.

\section{B. Lyapunov exponent}

Localized states are characterized by the localization length $\xi$. Here we introduce the Lyapunov exponent $\gamma$ which is the reciprocal of the localization length, i.e., $\gamma=1 / \xi$. The Lyapunov exponent $\gamma$ is defined by the ensemble average of logarithm of transmission rates [27],

$$
\gamma=-\lim _{L \rightarrow \infty} \frac{1}{2 L}\langle\ln T(L, \omega)\rangle \text {. }
$$

Figure 5 shows $\gamma$ versus frequency for the four pairs of incident angles of phonons corresponding with Fig. 2. $\gamma$ shows peaks and valleys with increasing frequency for the normal incidence of phonons in Fig. 5(a), and the peaks reach approximately $1.1 \mu \mathrm{m}^{-1}$ for the LA phonons and $1.3 \mu \mathrm{m}^{-1}$ for the TA phonons. At $\theta_{S, L}=15.0^{\circ}$ or $\theta_{S, T}=8.6^{\circ}$, the peaks of $\gamma$ become smaller than those of the normal incidence and are $0.8 \mu \mathrm{m}^{-1}$ for the LA phonon incidence and $0.9 \mu \mathrm{m}^{-1}$ for the TA phonon incidence as shown in Fig. 5(b). At the incident angles, the peaks of $\gamma$ are smaller than those of the normal incidence of phonons in the entire frequency region. In a finite system shorter than the localization length, i.e., $L \gamma \leqslant 1$, phonons are regarded to be extended. Supposing the RSL with $L=1 \mu \mathrm{m}$ for instance, phonons with $\gamma \leqslant 1 \mu \mathrm{m}^{-1}$ 

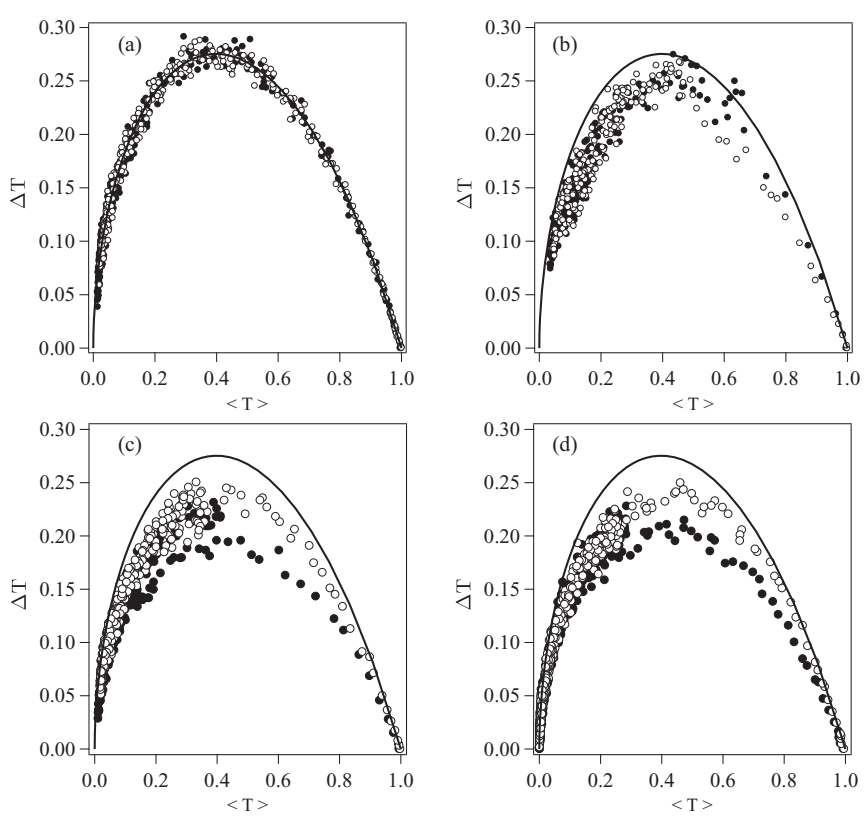

FIG. 6. The transmission fluctuations $\Delta T$ versus the averaged transmission rates $\langle T\rangle$ for four incident angles (a)-(d) corresponding to Fig. 2. The data are obtained by statistical evaluations of transmission rates of 300 samples of the RSL's composed of $N=1000$ layers. The open (solid) circles indicate $\Delta T$ for the LA(TA) incident phonons. The black solid lines are the theoretical $\Delta T$ versus $\langle T\rangle$ derived for single modes.

are extended. Then all the phonons in the entire frequency region are extended in the case of Fig. 5(b). The delocalization of phonons for the oblique phonon incidence is owing to the collaborations of phonons as mentioned above.

At $\theta_{S, L}=30.0^{\circ}$ and $\theta_{S, T}=16.9^{\circ}$, the Lyapunov exponents gather and exhibit similar frequency dependence as the transmission rates do. They coincide in particular at the incident angles $\theta_{S, L}=45.0^{\circ}$ or $\theta_{S, T}=24.2^{\circ}$ as shown in Fig. 5(d). The magnitude of $\gamma$ becomes larger than that of Figs. 5(b) and 5(c), and the peaks reach $\gamma \approx 2.5 \mu \mathrm{m}^{-1}$. It is almost twice as large as that of Fig. 5(c). Thus, the coupled modes induced by the mode conversions exhibit strong localization with increasing incident angles.

\section{Transmission fluctuations}

Because phonon propagation in the RSL's is owing to interference among multiply scattered phonons, the transmission rates fluctuate, depending on the realizations of the random order of unit layers. It is known for the single mode phonon transmission that the fluctuations defined by $\Delta T=$ $\sqrt{\left\langle T^{2}\right\rangle-\langle T\rangle^{2}}$ depends only on $\langle T\rangle$. The relation between $\Delta T$ and $\langle T\rangle$ is analytically proved to be a universal relation between them, independent of types of randomness [15].

Figure 6(a) shows $\Delta T$ versus $\langle T\rangle$ for the normal incidence of LA phonons (open circles) and that of TA phonons (solid circles) together with the theoretical results (solid line) for single mode phonons [15]. It is found that $\Delta T$ are small when $\langle T\rangle \sim 1$ and $\langle T\rangle \sim 0$, whereas $\Delta T$ are large for intermediate magnitudes of $\langle T\rangle$. Since the mode conversions do not occur for the normal phonon incidence, the numerical data for the
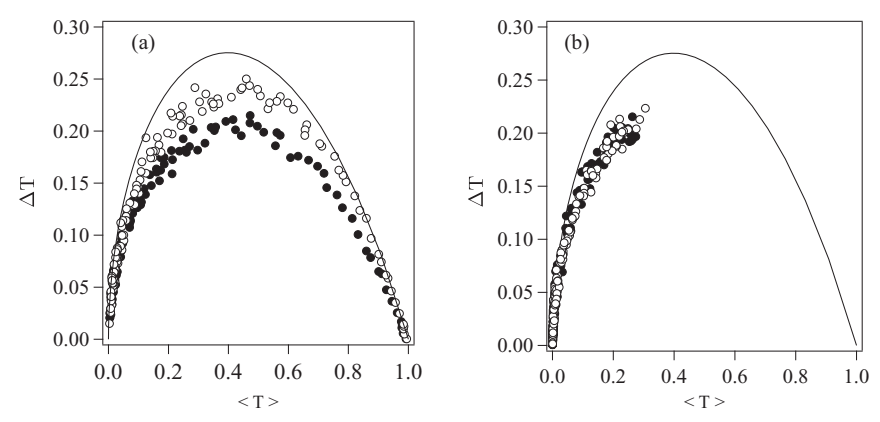

FIG. 7. The transmission fluctuations $\Delta T$ versus the averaged transmission rates $\langle T\rangle$ at $\theta_{S, L}=45^{\circ}$ and $\theta_{S, T}=24.2^{\circ}$ in the frequency region (a) $v \leqslant 0.2 \mathrm{THz}$ and (b) $0.2 \mathrm{THz} \leqslant v \leqslant 1 \mathrm{THz}$. The open (solid) circles indicate $\Delta T$ for the LA(TA) incident phonons. The black solid lines are the theoretical $\Delta T$ versus $\langle T\rangle$ derived for single modes. Figure 6(d) is composed of Figs. 7(a) and 7(b).

LA phonons and TA ones converge on the theoretical curve. Supposing that $\Delta T$ for the oblique incident phonons depends only on $\langle T\rangle$ like the normal incident phonons, we plot $\Delta T$ versus $\langle T\rangle$ in Figs. 6(b)-6(d) together with the theoretical results for single mode phonons. The incident angles are the same as those in Fig. 2.

As immediately found in Figs. 6(b)-6(d), $\Delta T$ for oblique phonon incidences are smaller than the theoretical curve for the normal incident phonons, meaning that the transmission fluctuations are suppressed. In addition, Figs. 6(c) and 6(d) exhibit complicated distributions of $\Delta T$. At first, we find the two arc-shaped distributions of $\Delta T$ similar to the theoretical curve for the normal incidence. It is also found that $\Delta T$ distribute between the two arcs in the region of $\langle T\rangle<0.4$ for Fig. 6(c) and $\langle T\rangle<0.3$ for Fig. 6(d). The arc-shaped distributions appear continuously from the extended states to the localized states, and the smooth variation is possible only in the low frequency region as understood from Figs. 2(c) and 2(d). On the other hand, the latter ones appear only for the localized states in the high frequency region. Then we divide the data in Fig. 6(d) at $0.2 \mathrm{THz}$ and plot individually $\Delta T$ versus $\langle T\rangle$ for (a) $v<0.2 \mathrm{THz}$ and for (b) $0.2 \mathrm{THz}<v<1.0 \mathrm{THz}$ in Fig. 7. Although we attributed above the phonon transmission to the phonons of coupled modes, Fig. 7(a) indicates that the phonon transmission in the low frequency region looks owing to the weakly linked single modes. Meanwhile the coupled mode phonons contribute in the high frequency region as confirmed in Fig. 7(b).

In order to confirm that the phonon transmission is owing to the single mode phonons in the low frequency region, we separate $\langle T\rangle$ for the RSL's examined in Fig. 2(d) into the compositions due to the transmitted phonons of LA and TA modes for the incident LA and TA phonons below $0.3 \mathrm{THz}$ in Fig. 8. The predominant transmitted phonon modes are obviously the same as the incident phonon modes below $0.1 \mathrm{THz}$, indicating that the phonons propagate as the single modes at low frequencies. Since the wavelengths are larger than $d_{A, B}$ in the frequency region, the phonon scattering is owing to the Rayleigh scattering. In the case of normal phonon incidence [15], the backscattering rate of phonons is proportional to $v^{2}$ and then the reflection in the frequency region is very small. Supposing this is the case for the oblique incident phonons, 


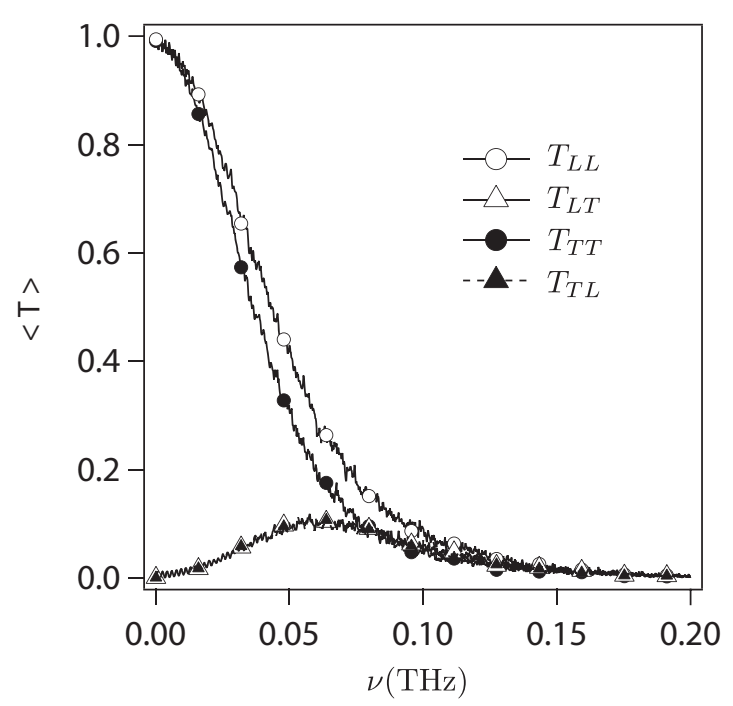

FIG. 8. The LA and TA components of $\langle T\rangle$ in the RSL's of $N=$ 1000 versus frequency. The incident angles are the same as Fig. 2(d). The solid lines with open or solid circles indicate the transmission rates of the same modes as the incident ones. The solid lines with open or solid triangles show the components of the different mode phonons from the incident phonons.

the mode coupling due to the encounters of transmitted and backscattered phonons is insufficient to induce the coupled modes and the incident phonons will remain as the predominant phonons. Thus we can consider that the transient in phonon modes from the single modes to the coupled ones is caused by the change in the scattering strength with frequency.

Before proceeding to investigate the origin of suppression of $\Delta T$, we briefly review the statistics on the transmission fluctuations of single mode phonons [15,28]. The transmission fluctuations for single mode phonons are related to the following probability density $W(z, t)$ with which one can express the average of an arbitrary function $f(z)$ of $z=1 / T$ as

$$
\langle f\rangle=\int_{1}^{\infty} f(z) W(z, t) d z .
$$

The probability density yields

$$
W(z, t)=\frac{2}{\sqrt{\pi t^{3}}} \int_{x_{0}}^{\infty} \frac{x}{\sqrt{\cosh ^{2} x-z}} e^{-\left(\frac{t}{4}+\frac{x^{2}}{t}\right)} d x
$$

where $t=\langle-\ln T\rangle=2 L \gamma$ and $x_{0}=\cosh ^{-1} \sqrt{z}$. For $t \gg 1$, the probability density is proved to be the log-normal distribution of transmission rates [28]

$$
W(z, t) z=\frac{1}{2 \sqrt{\pi t}} \exp \left[-\frac{(\ln z-t)^{2}}{4 t}\right] .
$$

$W(z, t) z$ becomes the probability density for $\ln z$ since $W(z, t) d z=W(z, t) z d(\ln z)$. It is apparent that the parameter $t$ gives the peak position of Eq. (29) and the variance of $\ln z$ as $(\Delta \ln z)^{2}=2 t$.

Here we investigate the phonon transmission at very small incident angles where the mode-conversion effects on the phonon propagation are very little as shown in Fig. 3. The incident phonons will remain largely unchanged, and a very small portion of the energy of incident phonons is converted
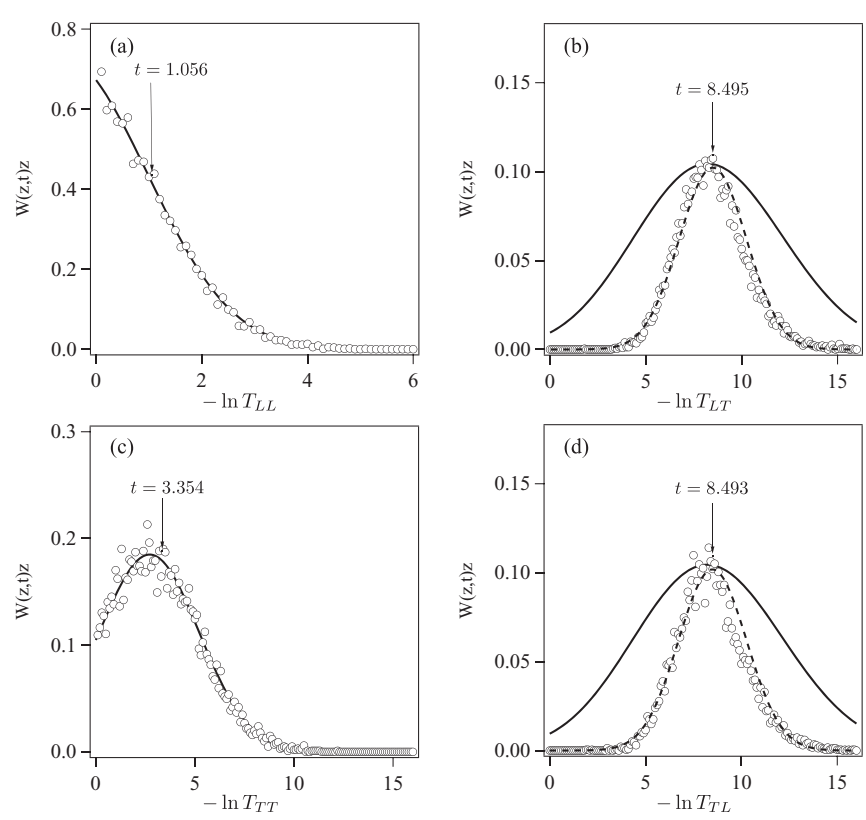

FIG. 9. The probability distributions of $-\ln T_{l m}$ at $v=0.1 \mathrm{THz}$. The incident angles are $\theta_{L}=1^{\circ}$ for LA phonons and $\theta_{T}=0.58^{\circ}$ for TA phonons. The open circles are the numerical data for 10000 samples, and the solid lines indicate the theoretical distribution estimated with Eq. (28). The arrows indicate the averaged magnitude $t$ of the numerical data. From the standard deviation of numerical data, we have $t_{L T}=1.547$ and $t_{T L}=1.586$, which are smaller than $t=8.495$ and $t=8.493$. The dashed lines indicate the revised probability distributions Eq. (30).

to the countermode phonons. Here we can ignore further mode conversions since the phonon intensity of the successive occurrences of reflectance is proportional to the product of $R_{L T}$ and $R_{T L}$. As a consequence, we may approximate the transmission rates to be composed of those of single mode phonons. Then we expect that the transmission rates of incident phonons $T_{L L}$ and $T_{T T}$ and those of mode-converted phonons $T_{L T}$ and $T_{T L}$ as well as their fluctuations are essentially the same as those of single mode phonons.

Figure 9 shows the probability distributions of the transmission fluctuations at $v=0.1 \mathrm{THz}$ of (a) $T_{L L}$, (b) $T_{L T}$, (c) $T_{T T}$, and (d) $T_{T L}$. The incident angles are $\theta_{L}=1^{\circ}$ for LA phonons and $\theta_{T}=0.58^{\circ}$ for TA phonons. The averaged transmission rates are $\left\langle T_{L L}\right\rangle=0.452$ and $\left\langle T_{T T}\right\rangle=0.189$, while $\left\langle T_{L T}\right\rangle$ and $\left\langle T_{T L}\right\rangle \approx 8 \times 10^{-4}$. The abscissa is $-\ln T_{l m}$ and the ordinate is the probability distribution. The solid lines indicate the theoretical probability distribution $W(z, t) z$ calculated from Eq. (28) for single mode phonons, where the parameter $t$ for $W(z, t) z$ in each case is estimated by averaging the logarithm of numerical data (open circles) of transmission rates as $t=-\left\langle\ln T_{l m}\right\rangle$. Obviously Figs. 9(a) and 9(c) show excellent agreement of the numerical data (open circles) with the theoretical results for the single mode phonons. The magnitudes of the numerical data are adjusted so that the peak heights of the data would match the theoretical ones. In contrast, although the peak positions of numerical data agree with those of theoretical curves for Figs. 9(b) and 9(d), the numerical data are distributed noticeably narrower than the theoretical curves. 
As shown in Eq. (29), the parameter $t$ determines both the peak position and the variance of the probability distribution. The disagreement about the variances found in Figs. 9(b) and 9(d) indicates that a single parameter is insufficient to express the probability distributions of phonons generated by mode conversions. Considering that the variance of transmission which is proportional to the Lyapunov exponent $\gamma$ is an intrinsic property of Anderson localization, we reexamine the relation of $t$ to the peak position of probability distribution.

As long as the detected phonon modes are the same, we may assume the same frequency dependence for $T_{L L}$ and $T_{T L}$ as the LA phonons and for $T_{T T}$ and $T_{L T}$ as the TA phonons. Introducing positive coefficients $\alpha, \beta, \alpha^{\prime}$ and $\beta^{\prime}$ which are smaller than unity, we put $T_{L L}=\alpha T_{L}^{0}, T_{L T}=$ $\beta T_{T}^{0}, T_{T T}=\alpha^{\prime} T_{T}^{0}$, and $T_{T L}=\beta^{\prime} T_{L}^{0}$, where $T_{l}^{0}$ means the transmission rates of single $l$-mode phonons without mode conversions for normal incidence. For very small incident angles, $\alpha, \alpha^{\prime} \approx 1$. As for $T_{L T}$, we have $t=-\left\langle\ln T_{L T}\right\rangle=$ $-\ln \beta-\left\langle\ln T_{T}^{0}\right\rangle . T_{T}^{0}$ is essentially dependent on the product of the Lyapunov exponents and the system length $t=2 \gamma L=$ $-\left\langle\ln T_{T}^{0}\right\rangle$, and the magnitude is expected to be the same as $t$ obtained in Figs. 9(c). However, they do not match because the mode conversions take place through the RSL's and then the system length $L$ is not well defined. The actual magnitudes of $t_{L T}=-\left\langle\ln T_{T}^{0}\right\rangle$ and $t_{T L}=-\left\langle\ln T_{L}^{0}\right\rangle$ can be derived from the variance of the numerical data, and then we have $t_{L T}=1.547$ and $t_{T L}=1.586$ in the case of Figs. 9(b) and 9(d). Then $\beta$ and $\beta^{\prime}$ are obtained by the subtractions $-\ln \beta=t-t_{L T}=8.495-1.547=6.984$ and $-\ln \beta^{\prime}=t-t_{T L}=8.493-1.586=6.907$. From the considerations, the probability distribution for $T_{L T}$ has variance given by $t_{L T}$ and the peak position shifts by $-\ln \beta$ from $t_{L T}$. We rewrite accordingly Eq. (29) for $T_{L T}$ as

$$
W(z, t) z=\frac{1}{2 \sqrt{\pi t_{L T}}} \exp \left[-\frac{\left(\ln z-\ln \beta-t_{L T}\right)^{2}}{4 t_{L T}}\right] .
$$

We have the same probability distribution as Eq. (30) for $T_{T L}$ where $t_{L T}$ and $\beta$ are replaced by $t_{T L}$ and $\beta^{\prime}$. The revised probability distribution curves are plotted with the dashed lines in Figs. 9(b) and 9(d), showing substantial agreement with the numerical data for both cases. Thus, the disagreement about the variances for $T_{L T}$ and $T_{T L}$ is owing not to the change in essential properties of Anderson localization but to the change in phonon intensities caused by mode conversions.

For the small incident angles discussed above, the suppression of transmission fluctuations of mode-converted phonons does not affect the statistics of the total transmission rates. With increasing incident angles, $R_{L T}$ and $R_{T L}$ become large, so that $\alpha$ and $\alpha^{\prime}$ decrease while $\beta$ and $\beta^{\prime}$ increase. The probability distributions of both incident phonons and modeconverted phonons will shift as discussed above, leading to the suppression of transmission fluctuations of them. Figure 10 shows the probability distributions of (a) $-\ln T_{L L}$, (b) $-\ln T_{L T}$, (c) $-\ln T_{T T}$, and (d) $-\ln T_{T L}$. The solid lines are the theoretical curves, and the open circles indicate the numerical results for 10000 samples. The parameter $t$ for each case is evaluated from the ensemble average of numerical data by $t=\left\langle-\ln T_{l m}\right\rangle$. The distributions of all the numerical data are found to be narrower than the theoretical ones.
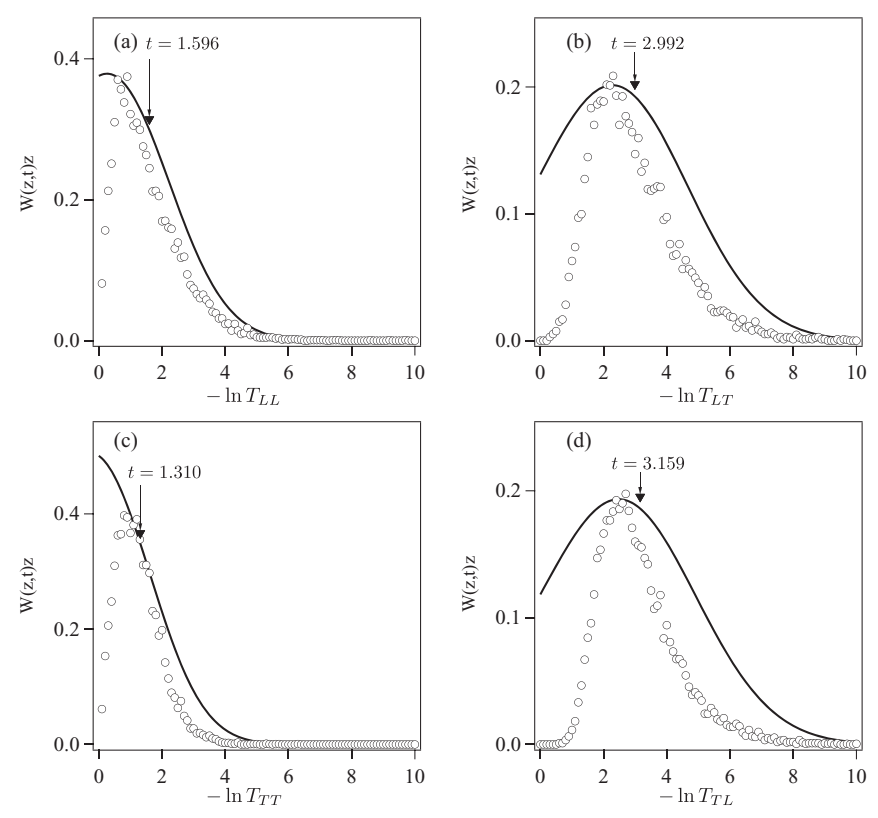

FIG. 10. The probability distributions at $v=0.0912 \mathrm{THz}$ for (a) $-\ln T_{L L}$ and (b) $-\ln T_{L T}$ and those at $v=0.0552 \mathrm{THz}$ for (c) $-\ln T_{T T}$ and (d) $-\ln T_{T L}$. The incident angles are $\theta_{L}=30^{\circ}$ for LA phonons and $\theta_{T}=16.9^{\circ}$ for TA phonons. The open circles are the numerical data for 10000 samples, and the arrows indicate the averaged magnitude $t$ of the numerical data. The averaged transmission rates are for each case (a) $\left\langle T_{L L}\right\rangle=0.296$, (b) $\left\langle T_{L T}\right\rangle=$ 0.0955 , (c) $\left\langle T_{T T}\right\rangle=0.332$, and (d) $\left\langle T_{T L}\right\rangle=0.0728$. The averaged total transmission rates are $\left\langle T_{L}\right\rangle=0.392$ and $\left\langle T_{T}\right\rangle=0.405$. The solid lines indicate the theoretical probability distributions Eq. (28).

Figure 11 shows the probability distributions of the total transmission fluctuations of Fig. 10. The averaged transmission rates of Figs. 11(a) and 11(b) are the same $\langle T\rangle \approx 0.4$. Comparing the two cases, we find that the probability distribution for the TA phonon incidence is suppressed more than that for the LA phonon incidence. The substantial suppression occurs at high transmission rates in particular for $T_{T}$. The difference between Figs. 11(a) and 11(b) stems from that the shift of the probability distribution of TA phonons shown in Fig. 10(c) is larger than that of LA phonons in Fig. 10(a).
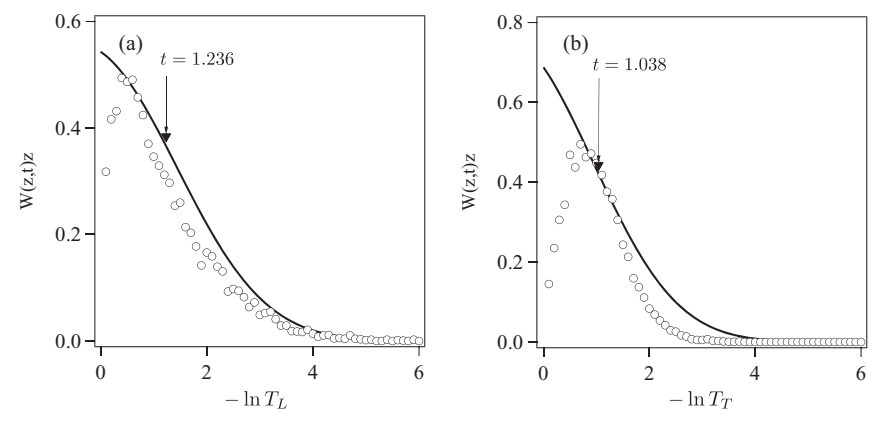

FIG. 11. The probability distribution of $-\ln T_{L}$ at $v=0.0912 \mathrm{THz}$ and that of $-\ln T_{T}$ at $v=0.0552 \mathrm{THz} . T_{L}$ and $T_{T}$ are the total transmission rates of Fig. 10. The open circles are the numerical data for 10000 samples, and the arrows indicate the averaged magnitude $t$ of the numerical data. The solid lines are the theoretical probability distributions Eq. (28). 

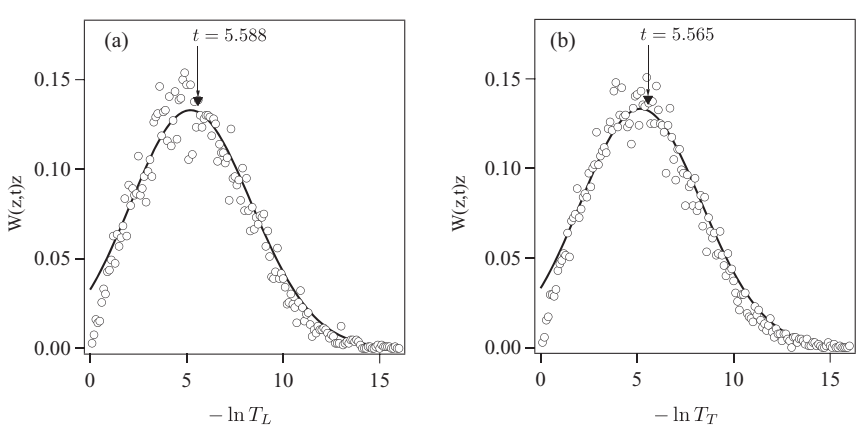

FIG. 12. The probability distributions of (a) $-\ln T_{L}$ and (b) $-\ln T_{T}$ at $v=0.4 \mathrm{THz}$. The incident angles are (a) $\theta_{L}=30^{\circ}$ for LA phonons and (b) $\theta_{T}=16.9^{\circ}$ for TA phonons. The open circles are the numerical results for 10000 samples, and the arrows indicate the averaged magnitude $t$ of the numerical data. The difference between the $t$ 's is quite negligible since the ratio of the difference in $t$ versus the standard deviation becomes $\frac{\Delta t}{\sigma} \approx 0.006$. Accordingly the averaged transmission rates are (a) $\left\langle T_{L}\right\rangle=0.039$ and (b) $\left\langle T_{T}\right\rangle=$ 0.040 . The numerical data also show excellent agreement with the theoretical probability distributions (solid lines) Eq. (28).

As a result, the transmission fluctuations of TA phonons are suppressed more than those of LA phonons as found in Fig. 6.

We finally investigate the probability distributions at $v=$ $0.4 \mathrm{THz}$ for $\theta_{L}=30^{\circ}$ and $\theta_{T}=16.9^{\circ}$ where the coupled phonon modes are expected. Figure 12 shows the numerical data for (a) LA phonon incidence and (b) TA phonon incidence together with the theoretical curves. The results are independent of the incident phonon modes like the averaged transmission rates in Fig. 2(c) and the Lyapunov exponents in Fig. 5(c). In contrast to Fig. 11, the numerical data show excellent agreement with the theoretical probability distribution of single mode phonons, which evidences the formation of coupled modes in the RSL's. The deviations of the numerical data from the theoretical curves are found at large transmission rates. This can be attributed to that there are no phonons simultaneously satisfying the resonant transmission conditions of LA and TA phonons in the RSL's.

\section{SUMMARY AND DISCUSSIONS}

We investigated the mode-conversion effects on Anderson localization of phonons in GaAs/AlAs random superlattices with the transfer matrix method, and made it clear what happens when the LA and TA phonons that independently show Anderson localization for normal incidence interact. For oblique phonon incidence, the mode conversions between the LA and TA phonons occur and the coupling becomes stronger with increasing incident angles. We revealed the characteristics brought by the mode conversions, paying attention to the change in the incident phonon mode dependences of transmission rates and the relation between the averaged transmission rates and transmission fluctuations.

One of the interesting findings of the present work is weakening localization properties. The mode conversions induce the collaborations among the phonons at small incident angles that compensate for the transmission decay. As a result, the localization lengths are extended, and then the phonons are possibly delocalized, depending on the system size. The transmission fluctuations are suppressed in comparison with those of single modes phonons. The disagreement is not owing to the change in intrinsic statistics of transmission fluctuations but stems from the energy sharing of phonons due to the mode conversions.

At larger incident angles, the mode conversions become strong enough to form the coupled modes peculiar to the system whose transmission rates and transmission fluctuations are independent of the incident phonon modes. The agreement of transmission fluctuations of the coupled mode phonon with the single mode phonons confirms the formation of single mode phonons.

To conclude, the presence of mode conversions does not violate the localization phenomena but gives rise to a variety of phenomena owing to interaction among phonons.

\section{APPENDIX: MATRIX ELEMENTS}

The matrix elements of $\Lambda_{n}\left(d_{n}\right)$ are given as

$$
\begin{aligned}
& \lambda_{11}^{n}\left(d_{n}\right) \equiv e^{i q_{n, L} d_{n}} \cos \theta_{n, L} \\
& \lambda_{12}^{n}\left(d_{n}\right) \equiv-e^{-i q_{n, L} d_{n}} \cos \theta_{n, L} \\
& \lambda_{13}^{n}\left(d_{n}\right) \equiv-e^{i q_{n, T} d_{n}} \sin \theta_{n, T} \\
& \lambda_{14}^{n}\left(d_{n}\right) \equiv e^{-i q_{n, T} d_{n}} \sin \theta_{n, T} \\
& \lambda_{21}^{n}\left(d_{n}\right) \equiv e^{i q_{n, L} d_{n}} \sin \theta_{n, L} \\
& \lambda_{22}^{n}\left(d_{n}\right) \equiv e^{-i q_{n, L} d_{n}} \sin \theta_{n, L} \\
& \lambda_{23}^{n}\left(d_{n}\right) \equiv e^{i q_{n, T} d_{n}} \cos \theta_{n, T} \\
& \lambda_{24}^{n}\left(d_{n}\right) \equiv e^{-i q_{n, T} d_{n}} \cos \theta_{n, T} \\
& \lambda_{31}^{n}\left(d_{n}\right) \equiv \frac{\left(C_{n, 11} \cos ^{2} \theta_{n, L}+C_{n, 12} \sin ^{2} \theta_{n, L}\right)}{c_{n, L}} e^{i q_{n, L} d_{n}} \\
& \lambda_{32}^{n}\left(d_{n}\right) \equiv \frac{\left(C_{n, 11} \cos ^{2} \theta_{n, L}+C_{n, 12} \sin ^{2} \theta_{n, L}\right)}{c_{n, L}} e^{-i q_{n, L} d_{n}} \\
& \lambda_{33}^{n}\left(d_{n}\right) \equiv-\frac{\left(C_{n, 11}-C_{n, 12}\right) \sin \theta_{n, T} \cos \theta_{n, T}}{c_{n, T}} e^{i q_{n, T} d_{n}} \\
& \lambda_{34}^{n}\left(d_{n}\right) \equiv-\frac{\left(C_{n, 11}-C_{n, 12}\right) \sin \theta_{n, T} \cos \theta_{n, T}}{c_{n, T}} e^{-i q_{n, T} d_{n}} \\
& \lambda_{41}^{n}\left(d_{n}\right) \equiv \frac{C_{n, 44} \sin 2 \theta_{n, L}}{c_{n, L}} e^{i q_{n, L} d_{n}} \\
& \lambda_{42}^{n}\left(d_{n}\right) \equiv-\frac{C_{n, 44} \sin 2 \theta_{n, L}}{c_{n, L}} e^{-i q_{n, L} d_{n}} \\
& \lambda_{43}^{n}\left(d_{n}\right) \equiv \frac{C_{n, 44} \cos 2 \theta_{n, T}}{c_{n, T}} e^{i q_{n, T} d_{n}} \\
& \lambda_{44}^{n}\left(d_{n}\right) \equiv-\frac{C_{n, 44} \cos 2 \theta_{n, T}}{c_{n, T}} e^{-i q_{n, T} d_{n}} .
\end{aligned}
$$

The matrix elements of $\Lambda_{S}$ and $\Lambda_{D}$ are derived from the above expressions by putting $d_{n}=0$ and substituting the elastic constants by those of the $\mathrm{S}$ and $\mathrm{D}$ layers, and $\theta_{n,\{L, T\}}$ is also substituted by $\theta_{S,\{L, T\}}$ for $\Lambda_{S}$ or by $\theta_{D,\{L, T\}}$ for $\Lambda_{D}$. 
[1] B. Souillard, in Chance and Matter, edited by J. Souletie, J. Vannimenus, and R. Stora (North-Holland, Amsterdam, 1987), p. 305.

[2] S. John, in Scattering and Localization of Classical Waves in Random Media, edited by P. Sheng (World Scientific, Singapore, 1990), p. 1.

[3] S. John, H. Sompolinsky, and M. J. Stephen, Phys. Rev. B 27, 5592 (1983).

[4] T. R. Kirkpatrick, Phys. Rev. B 31, 5746 (1985).

[5] S. John, Phys. Rev. Lett. 58, 2486 (1987).

[6] R. Venkatasubramanian, E. Siivola, T. Colpitts, and B. O'Quinn, Nature (London) 413, 597 (2001).

[7] C. Monthus and T. Garel, Phys. Rev. B 81, 224208 (2010).

[8] J. Ravichandran, A. K. Yadav, R. Cheaito, P. B. Rossen, A. Soukiassian, S. J. Suresha, J. C. Duda, B. M. Foley, C.-H. Lee, Y. Zhu, A. W. Lichtenberger, J. E. Moore, D. A. Muller, D. G. Schlom, P. E. Hopkins, A. Majumdar, R. Ramesh, and M. A. Zurbuchen, Nat. Mater. 13, 168 (2014).

[9] K. Ishii, Prog. Theor. Phys. Suppl. 53, 77 (1973).

[10] T. Juntunen, O. Vänskä, and I. Tittonen, Phys. Rev. Lett. 122, 105901 (2019).

[11] S. D. Pinski, W. Schirmacher, and R. A. Römer, Europhys. Lett. 97, 16007 (2012).

[12] H. Yamada, J. Phys. Soc. Jon. 72, 125 (2003).

[13] E. Abrahams, P. W. Anderson, D. C. Licciardello, and T. V. Ramakrishnan, Phys. Rev. Lett. 42, 673 (1979).
[14] S. Tamura and F. Nori, Phys. Rev. B 41, 7941 (1990).

[15] N. Nishiguchi, S. Tamura, and F. Nori, Phys. Rev. B 48, 2515 (1993).

[16] N. Nishiguchi, S. Tamura, and F. Nori, Phys. Rev. B 48, 14426 (1993).

[17] A. Chaudhuri, A. Kundu, D. Roy, A. Dhar, J. L. Lebowitz, and H. Spohn, Phys. Rev. B 81, 064301 (2010).

[18] H. A. Fertig and T. L. Reinecke, Phys. Rev. B 50, 7443 (1994).

[19] J. Mendoza and G. Chen, Nano Lett. 16, 7616 (2016).

[20] M. N. Luckyanova, J. Mendoza, H. Lu, B. Song, S. Huang, J. Zhou, M. Li, Y. Dong, H. Zhou, J. Garlow, L. Wu, B. J. Kirby, A. J. Grutter, A. A. Puretzky, Y. Zhu, M. S. Dresselhaus, A. Gossard, and G. Chen, Sci. Adv. 4, eaat9460 (2018).

[21] P. Chakraborty, L. Cao, and Y. Wang, Sci. Rep. 7, 8134 (2017).

[22] L. R. Weisberg and J. Blanc, J. Appl. Phys. 34, 1002 (1963).

[23] T. B. Bateman, H. J. McSkimin, and J. M. Whelan, J. Appl. Phys. 30, 544 (1959).

[24] J. D. Wiley, Semiconductors and Semimetals (Academic, New York, 1975), Vol. 10, p. 115.

[25] S. Adachi, J. Appl. Phys. 58, R1 (1985).

[26] S. Tamura and J. P. Wolfe, Phys. Rev. B 35, 2528 (1987).

[27] I. M. Lifshitz, S. A. Gredeskul, L. A. Pastur, and E. Yankovsky, Introduction to the Theory of Disordered Systems (Wiley, New York, 1988).

[28] A. A. Abrikosov, Solid State Commun. 37, 997 (1981). 